\title{
Canonical correlations between individual self-efficacy/organizational bottom-up approach and perceived barriers to reporting medication errors: a multicenter study
}

Myoung Soo Kim ${ }^{1}$ and Chul-Hoon $\mathrm{Kim}^{2^{*}}$

\begin{abstract}
Background: Individual and organizational factors correlate with perceived barriers to error reporting. Understanding medication administration errors (MAEs) reduces confusion about error definitions, raises perceptions of MAEs, and allows healthcare providers to report perceived and identified errors more frequently. Therefore, an emphasis must be placed on medication competence, including medication administration knowledge and decision-making. It can be helpful to utilize an organizational approach, such as collaboration between nurses and physicians, but this type of approach is difficult to establish and maintain because patient-safety culture starts at the highest levels of the healthcare organization. This study aimed to examine the canonical correlations of an individual self-efficacy/bottomup organizational approach variable set with perceived barriers to reporting MAEs among nurses.
\end{abstract}

Methods: We surveyed 218 staff nurses in Korea. The measurement tools included a questionnaire on knowledge of high-alert medication, nursing decision-making, nurse-physician collaboration satisfaction, and barriers to reporting MAEs. Descriptive statistics, t-tests, analysis of variance (ANOVA), Pearson's correlation coefficient, and canonical correlations were used to analyze results.

Results: Two canonical variables were significant. The first variate indicated that less knowledge about medication administration $(-0.83)$ and a higher perception of nurse-physician collaboration $(0.42)$ were related to higher disagreement over medication error (0.64). The second variate showed that intuitive clinical decision-making (- 0.57) and a higher perception of nurse-physician collaboration (0.84) were related to lower perceived barriers to reporting MAEs.

Conclusions: Enhancing positive collaboration among healthcare professionals and promoting analytic decision-making supported by sufficient knowledge could facilitate MAE reporting by nurses. In the clinical phase, providing medication administration education and improving collaboration may reduce disagreement about the occurrence of errors and facilitate MAE reporting. In the policy phase, developing an evidence-based reporting system that informs analytic decision-making may reduce the perceived barriers to MAE reporting.

Keywords: Clinical decision-making, Intersectoral collaboration, Knowledge, Medication errors, Social perception

\footnotetext{
* Correspondence: bbp2000@dau.ac.kr

${ }^{2}$ College of Medicine, Dong-A University Hospital, 26 Daesingongwon-ro,

Seo-gu, Busan 49201, South Korea

Full list of author information is available at the end of the article
}

(c) The Author(s). 2019 Open Access This article is distributed under the terms of the Creative Commons Attribution 4.0 International License (http://creativecommons.org/licenses/by/4.0/), which permits unrestricted use, distribution, and reproduction in any medium, provided you give appropriate credit to the original author(s) and the source, provide a link to the Creative Commons license, and indicate if changes were made. The Creative Commons Public Domain Dedication waiver (http://creativecommons.org/publicdomain/zero/1.0/) applies to the data made available in this article, unless otherwise stated. 


\section{Background}

Patient-safety reporting systems have been used to detect the root causes of errors and to enhance patient safety and the economic efficiency of healthcare [1]. However, the number of errors reported by these means is less than the number of actual errors determined by experts [2]. Because under-reporting errors is a critical limitation to analyzing the process behind medication administration errors (MAEs) [3], understanding perceived barriers to error reporting is crucial in promoting reporting behavior. Several studies have shown individual and organizational factors for enhancing error- reporting [2, 3], and one study [4] proposed a conceptual model for determinants of MAE reporting with an individual perceived self-efficacy and organizational factors such as reporting process capability and support, organizational culture, management support, and regulatory authority.

Individual perceived self-efficacy is defined as an individual's ability or competence to effectively cope with responding to MAEs [4]. Understanding MAEs reduces confusion about error definitions [5], raises levels of clinical reasoning about whether the error was harmful [6], and allows more frequent reporting of perceived and identified errors by healthcare providers [5]. Therefore, required competencies for medication safety were a knowledge and decisionmaking [7]. Collection of audit data and reporting of errors provide evidence-based knowledge of medication administration standards [8]. However, the information accumulated to measure knowledge is insufficient, owing to the lack of feedback on error-reporting systems [8]. Furthermore, while the majority of MAEs do not result in harm [9], some drugs, known as 'high-alert' drugs, are more likely to cause significant patient harm when used in error [10]. More attention should be paid to substances categorized as high-alert drugs by the US Institute for Safe Medication Practices [10] and a systematic review [11], including anticoagulants, cardiovascular drugs, insulins, and opiates, among others. Specific knowledge about high-alert medication, which is high -risk medication for nurses' MAEs [12], can serve as the basic competence level when judging what is an MAE and deciding whether to report it.

Judging medication errors and the decision to report them may depend on nurses' knowledge base and their decision-making ability; however, there is insufficient previous research on nurses' decisions to report errors. In particular, barriers to reporting MAEs may depend on whether decision-making is analytic or intuitive [13]. According to analytical-intuitive decision-making theory [14], cognitive processes may move along the analyticalintuitive continuum over time. Generally, analytical decision-making is known as an appropriate decisionmaking style when information is organized in memory and the correct weight and importance is given to each piece of information $[14,15]$. Intuitive decision-making is defined as an unconscious, not logically defensible, and non-stepwise process $[14,15]$. However, decisionmaking cannot be the same across diverse nursing situations [15], and little is known about the relationship between nurses' decision-making and error- reporting situations.

Regarding organizational factors, improving patientsafety culture and supportive leadership is a key organization-based strategy for enhancing error reporting $[4,16]$. Safety culture has major effects on patient outcomes and a positive safety culture in healthcare is evidenced by the visibility of leaders and support for patient-safety initiatives [17]. However, because patient-safety culture starts at the highest levels of the healthcare organization [16], it is difficult to establish and maintain supportive management of medication safety [18]. Therefore, it can be helpful to utilize a bottom-up approach [19]. A bottom-up approach to improving patient-safety culture means that patientsafety awareness should be embedded at all staff levels and be seen in all actions [20], and collaboration among healthcare providers can establish effective communication [21] and create a positive patient-safety culture. Communication openness regarding mistakes and failures is a crucial factor in patient-safety; however, communication remains an adaptive challenge that has proven difficult to overcome in the sociotechnical viewpoint that defines healthcare [22]. Most of the current literature has focused on a top-down approach such as supportive leadership for enhancing error reporting [16-18] and there has been limited research on bottom-up approaches such as collaboration.

Although several studies have demonstrated perceived barriers to error reporting, there is a gap in the empirical study of areas such as knowledge for errors, decisionmaking, and the organizational bottom-up approach. Furthermore, there may be combination effects of variables in relation to individual or organizational factors for perceived barriers to MAE reporting. Based on the fact that communication and pharmacological knowledge impact nurses' decision-making concerning medication [23], research variables are expected to show something other than a bivariate relationship with perceived barriers to MAE reporting. Therefore, the current research questions were set as follows: (1) is there a relationship between an individual self-efficacy/organizational bottom-up approach set and perceived barriers to MAE reporting? And if so, (2) what factor has the strongest relationship for reducing perceived barriers to reporting MAE?

\section{Methods}

\section{Study design and participants}

This study employed a cross-sectional descriptive design and non-probability sampling was used. The address book of the Korean Nurses Association was used to create a list of hospitals. We were able to obtain the telephone 
numbers and e-mail addresses of the nursing administration departments at each hospital. Two hundred and seventy four secondary and tertiary hospitals were invited to take part, of which 28 responded to the first e-mail. Finally, the department heads of seven hospitals gave us permission to distribute the questionnaire after reviewing the research protocol and the IRB's approval. The inclusion criteria for participating registered nurses (RNs) were a) having at least 3 months' clinical experience (because the first 3 months are generally a job-related orientation period in Korea) and b) not administrative nurses, such as unit managers or charge nurses, because they are more likely than junior staff nurses to know how or what to do about reporting [24], potentially biasing the results.

To control possible confounding variables of work experience, job location, and job position, we asked the nursing department to distribute anonymous selfadministered questionnaires to nurses in a minimum of three units in each hospital. Three to five units in each hospital were included in the data collection. A variety of wards (medical, surgical, rehabilitation, gynecology, pediatric) and intensive care units (medical, surgical, cardiac, neuroscience, traumatic) were included. Many hospitals limited the number of participants in order to minimize nurses' additional workload. Therefore, 20 questionnaires were provided for one hospital with less than 500 beds, 30 questionnaires for two hospitals with 500-699 beds, 45 questionnaires for two hospitals with 700-999 beds, and 50 questionnaires for two hospitals with more than 1000 beds. We hired one nurse as a research assistant in each hospital to distribute and collect the data. They distributed questionnaires to staff nurses at the direction of the nursing department. Respondents placed completed, sealed questionnaires in a box located at the nurse station on each floor and research assistants collected the questionnaires.

A total of 270 staff nurses were surveyed from November 2012 to December 2013. The mean duration of questionnaire distribution and collection was 2 months. However, two hospitals gave late permission for survey research on August 2013, so the duration of questionnaire distribution and collection was 3 months, from September to December 2013. Two -hundred thirtyone questionnaires were returned (response rate of 85.6\%). After excluding thirteen questionnaires whose respondents had less than 3 months of work experience (7) or had missing answers (6), 218 questionnaires were included in the analysis. The sample size was determined based on the recommendation that about ten cases are needed for every variable in the canonical correlation [25]. In this study, there are seven variables in the final analysis, so 218 participants would be acceptable.

\section{Outcome measures \\ Individual self-efficacy/organizational bottom-up approach set}

Individual self-efficacy; knowledge of medication administration High-alert medications may cause significant patient harm when used in error; however, highalert drugs have not been categorized in Korea. Therefore, potassium chloride, insulin, morphine, heparin, and warfarin drugs were regarded as high-alert drugs based on a specific research [10]. To examine knowledge of medication administration, we used a 20-item questionnaire about "knowledge of high-alert medication" developed by Hsiao et al. [12] and verified in Korean by Kim and Jung [26]. The questionnaire had two parts: Part A evaluated drug administration with a focus on drug delivery routes and dosage; and Part B evaluated drug regulations with a focus on how high-alert medications should be stored, regulated, and documented. Therefore, knowledge of high-alert medication administration is considered to contain valuable information about medication errors. Five-points were given for a correct answer, resulting in a total possible score range of 0-100. The KR-20 value, an assessment of reliability, estimates the consistency of measurement using the variance of item scores and is used when the items are dichotomous. It can also estimate consistent reliability compared to the split-half estimation. Internal consistency in the previously reported KR-20 [12] was 0.74 ; in a previous study [26] it was 0.61 , and in this study, it was .62, indicating relatively low reliability. However, a tentative social psychological instrument can be used for research if the reliability of the instrument is over .60 [27].

Individual self-efficacy; nursing decision-making A 24-item nursing decision-making instrument was used, developed by Lauri and Salanterä [15] and verified in Korean by Kim and Jung [28]. This instrument was developed with reference to evidence that nurses flexibly apply diverse kinds of decision-making models depending on the specific nursing problem, the task at hand, the information available, organization level, and time constraints [15]. The instrument consisted of 24 items scored from one (definitely disagree) to five (definitely agree). Scores for responses to odd items were reversed, and those for even items were not changed [15]. Scores were summed; a low score indicated an analytic approach to decision-making, a high score an intuitive approach [15]. Cronbach's alpha for this study was 0.87 .

An organizational bottom-up approach; nurse -physician collaboration satisfaction A nurse -physician collaboration satisfaction questionnaire [29] was used to investigate an organizational bottom-up approach. To 
assess semantic equivalence, translation and backtranslation using a synthesis of translations by two bilingual professionals were conducted. Content validity was checked by two experts, one nursing professor and one nursing unit manager with a doctoral degree. The content validity index for relevance and representativeness was calculated for each item using a 4-point scale $(4=$ highly relevant, 3 = quite relevant, $2=$ somewhat relevant, $1=$ not relevant). I-CVI (item CVI) is 1.00 , which is acceptable because Lynn's recommendation [30] is that I-CVI should be 1.00 when there are five or fewer experts. Based on the Polit's study [31], S-CVI (scale CVI) was valid because it is over 0.9. This instrument consisted of eight items with seven response options; six of the items ranged from "strongly disagree" to "strongly agree," one ranged from "no collaboration" to "complete collaboration," and one ranged from "not satisfied" to "very satisfied." Higher scores indicated greater collaboration satisfaction between nurses and physicians in an organization. Cronbach's alpha was 0.93 for this study.

\section{Perceived barriers to reporting MAEs}

The Barriers to Reporting MAEs instrument, which was developed by Wakefield, Wakefield, Uden-Holman and Blegen [32] and verified for semantic equivalence and content validity in Korean by the research team, was used. Two of the researchers translated the instrument into Korean using reverse-translation and bilingual techniques as suggested by Brislin [33]. I-CVI was 0.87 and S-CVI was 0.82 because the study included six experts who worked for the patient-safety departments of two hospitals. An exploratory factor analysis with varimax rotation was performed and items with component loadings greater than 0.3 were retained [25]. Factorability was examined using a Kaiser-Meyer-Olkin (KMO) test. Kaiser's measure of sampling adequacy requires a value of 0.6 or above [34]; our test gave a KMO of .91, indicating good factor analysis. Three dimensions indicated a poor fit of the model to our data; we therefore carried out analysis to determine a four-dimension structure. As a result, total percentage of variance was $61.8 \%$ and the 16 -item instrument included four dimensions (fear, disagreement over whether an error occurred, effort required to report MAEs, and administrative responses to medication errors). Higher scores corresponded to a higher perception of barriers to reporting MAE. Item responses were measured on a five-point agreement scale from one (definitely disagree) to five (definitely agree). Cronbach's alpha for this study was 0.76 , indicating that this instrument was suitable for a social psychological study.

\section{Ethical considerations}

This study was conducted after being reviewed and approved by the institutional review board (IRB) at one of the participant hospitals in this study (IRB no. E20120821). Several hospitals were unable to review and approve the study because they either did not have individual IRBs or did not have employees on the research team. Therefore, the research team recruited hospitals that gave their permission after reviewing the research protocol and the approval of one IRB. On the first page of the survey, the purpose, voluntary participation, confidentiality of the information, and procedures of the study were explained. Written consent was obtained from participants prior to questionnaire distribution.

\section{Data analysis}

Data were analyzed using SPSS 23.0 (SPSS, Inc., Chicago, IL. USA). Descriptive analyses, independent t-tests, and ANOVA were used. Pearson correlation was used for screening assumption; the results of the correlation coefficient values were not interpreted. Canonical correlation analysis was employed after the assumptions of the canonical correlation were checked. Interpretation of reliable pairs of canonical variates was based on the loading matrices. Because the loading matrices contained correlations, and squared correlations measure overlapping variances [25], variables with correlations of 0.30 and above were interpreted.

Internal consistency is usually calculated from the pairwise correlations between items and some social psychological instruments can have lower reliability [27]. Even though a commonly accepted rule of thumb for describing internal consistency is to deem it acceptable when it is between 0.7 and 0.8 , an internal consistency over 0.6 is acceptable in this study because a tentative social science instrument was included [27].

\section{Results}

\section{Perceived barriers to reporting MAE according to participants' characteristics}

The mean age of the participants was 28.6 years, and almost all participants (97.2\%) were female. Only $1.3 \%$ of the participants had a master's degree, while $76.6 \%$ were married. Twenty-eight percent of the participants had above 1 year and less than 3years of total nursing experience. There were no significant differences in perceived barriers to reporting MAEs; however, we observed a lower average rating for perceived barriers to reporting MAEs among participants with a master's degree $(\mathrm{F}=4.13, p=.017)$ (Table 1$)$.

\section{Knowledge about medication administration, nursing decision-making, nurse-physician collaboration satisfaction, and perceived barriers to reporting MAE}

The correct-answer rate for knowledge of medication administration was $65 \%$. The nursing decision-making mean score was $2.93 \pm 0.11$. The overall mean score of nursephysician collaboration satisfaction was $4.01 \pm 0.97$. The 
Table 1 Perceived Barriers to Reporting Medication Administration Errors according to Socio-demographic Characteristics of Participants

\begin{tabular}{|c|c|c|c|c|}
\hline \multirow[t]{3}{*}{ Characteristics } & & \multirow[t]{3}{*}{$n(\%)$} & \multicolumn{2}{|r|}{$(N=218)$} \\
\hline & & & \multicolumn{2}{|c|}{ Perceived barriers to reporting medication administration erro } \\
\hline & & & $\bar{M} \pm S D$ & t or $F(p)$ \\
\hline Age & $22-29$ & $161(65.2)$ & $2.71 \pm 0.42$ & 2.65(.073) \\
\hline \multirow[t]{2}{*}{$(28.60 \pm 6.15)$} & $30-39$ & 48(25.9) & $2.56 \pm 0.38$ & \\
\hline & Above 40 & $9(8.9)$ & $2.72 \pm 0.55$ & \\
\hline \multirow[t]{2}{*}{ Gender } & Male & $6(2.8)$ & $2.38 \pm 0.62$ & $-1.82(.070)$ \\
\hline & Female & 212(97.2) & $2.69 \pm 0.41$ & \\
\hline \multirow[t]{3}{*}{ Education level } & College & 93(42.7) & $2.64 \pm 0.41$ & 4.13(.017) \\
\hline & University & 122(56.0) & $2.72 \pm 0.41$ & \\
\hline & Master's degree & $3(1.3)$ & $2.08 \pm 0.67$ & \\
\hline \multirow[t]{2}{*}{ Marital status } & Single & $51(23.4)$ & $2.61 \pm 0.46$ & $-1.39(.165)$ \\
\hline & Married & 167(76.6) & $2.70 \pm 0.40$ & \\
\hline \multirow[t]{6}{*}{ Total experience in nursing (year) } & $<1$ & $37(17.0)$ & $2.66 \pm 0.43$ & $0.17(.975)$ \\
\hline & $1-<3$ & $61(28.0)$ & $2.67 \pm 0.42$ & \\
\hline & $3-<5$ & $48(22.0)$ & $2.72 \pm 0.41$ & \\
\hline & $5-<10$ & 45(20.6) & $2.67 \pm 0.41$ & \\
\hline & $10-<15$ & 18(8.3) & $2.64 \pm 0.40$ & \\
\hline & $\geq 15$ & $9(4.1)$ & $2.71 \pm 0.56$ & \\
\hline \multirow[t]{4}{*}{ Number of bed } & $<500$ & $13(6.0)$ & $2.70 \pm 0.50$ & $0.31(.818)$ \\
\hline & $500-<700$ & 34(15.6) & $2.66 \pm 0.37$ & \\
\hline & $700-<1000$ & $83(38.1)$ & $2.65 \pm 0.44$ & \\
\hline & $\geq 1000$ & $88(40.4)$ & $2.71 \pm 0.40$ & \\
\hline Total mean score & & & $2.66 \pm 0.41$ & \\
\hline
\end{tabular}

mean score of perceived barriers to reporting MAEs was $2.68 \pm 0.42$ (Table 2).

\section{Correlations between individual self-efficacy/ organizational bottom-up approach set and perceived barriers to reporting MAEs set}

The assumptions of canonical correlation with regard to normality, linearity, homoscedasticity, and multicollinearity were met. The correlations between perceived barriers to reporting MAEs variables were .20-.52, indicating no within-set multicollinearity. The correlation between individual self-efficacy/organizational bottomup variables and perceived barriers to reporting MAEs variables were $|.16|-|.24|$, which showed the absence of multicollinearity (Table 3).

There were two pairs of significant canonical variates. The first canonical correlation was 0.37 and the second was 0.23 . With all four canonical correlations included, Wilks' lambda was $0.61(F=4.19, p<.001)$; with the first canonical correlation removed, Wilks' lambda was 0.89 $(F=2.88, p=.009)$. The first pair of canonical variates indicated that those with lower knowledge of medication administration (- 0.83), and inclined toward intuitive decision -making (0.41), and higher nurse -physician collaboration satisfaction (0.42), were associated with a higher perception of disagreement of over whether an error had occurred (0.64). The second canonical variate suggested that a combination of analytic decision -making (- 0.57) and higher nurse -physician collaboration satisfaction (0.84) was associated with lower perception of administration responses $(-0.94)$, disagreement over medication error ($0.67)$, reporting effort $(-0.46)$, and fear $(-0.42)$ (Table 4$)$.

\section{Discussion}

The study aimed to define the roles of individual/ organizational factors in reducing perceived barriers to reporting MAEs using canonical correlations; therefore, the findings are discussed by focusing on the significant canonical variates among study variables.

We identified the perceived barriers to reporting MAEs according to the participants' characteristics. Nurses with a master's degree perceived lower barriers to reporting MAEs than the others, though it is hard to generalize the result because only three participants had a master's degree. Education was a barrier for reporting MAEs in a previous research [34]. According to one study, more 
Table 2 Knowledge about Medication Administration, Clinical Decision-Making, Nurse -physician Collaboration Satisfaction, and Perceived Barriers to Reporting Medication Administration Errors

\begin{tabular}{lll}
\hline Variables (number of items) & Mean \pm SD & $(N=218)$ \\
& & Actual range \\
\hline Knowledge about medication administration (20) & $0.65 \pm 0.15$ & $0.10 \sim 0.90$ \\
Clinical decision-making (24) & $2.93 \pm 0.11$ & $2.63 \sim 3.42$ \\
Nurse -physician collaboration satisfaction (8) & $4.01 \pm 0.97$ & $1.00 \sim 6.50$ \\
Perceived barriers to reporting medication administration error (16) & $2.68 \pm 0.42$ & $1.31 \sim 3.75$ \\
Fear (5) & $3.35 \pm 0.65$ & $1.60 \sim 5.00$ \\
Disagreement over medication error (5) & $2.17 \pm 0.53$ & $1.00 \sim 4.00$ \\
Reporting effort (2) & $2.86 \pm 0.73$ & $1.00 \sim 5.00$ \\
Administrative responses (4) & $2.96 \pm 0.59$ & $1.00 \sim 5.00$ \\
\hline
\end{tabular}

confident and autonomous and more experienced nurses might think that not all medication errors are severe enough to be reported [35]. However, because underreporting makes it impossible to accurately analyze the medication process behind errors or near misses, all errors, regardless of their severity, should be reported in order to ensure medication safety [2]. Emphasis on the benefits of reporting was provided to the nurses in continuing education or in their graduate coursework. To prove a stronger relationship, it is necessary to examine the relationship between educational status and barriers to MAE reporting after increasing the number of participants and to test the effectiveness of the educational program.

The highest perceived barrier to MAE reporting was fear, a result similar to a previous study [36]. Fear may come from Korean cultural attributes such as hierarchical culture and face-saving concern [36]. If nurses feel that they are working within a strongly hierarchical structure, they may be afraid of co-workers' and unit managers' potential reactions to MAE reporting, such as blame or punishment [6]. Face-saving is defined as an individual's claimed sense of positive feelings in the context of social interactions [37]. Health professionals in conservative and collectivistic cultures are probably reluctant to report coworkers' MAEs in order to maintain harmonious interrelationships [36]. This conservative culture and hierarchy may mean that trying to look for and report errors would be regarded as making their colleagues uncomfortable or even a betrayal. Therefore, chief executives in hospital organizations should try to build a non-punitive culture [38] and members of organizations need to think of reporting behavior as a way of improving patient-safety practice, not in terms of gaining or losing face.

There are two significant canonical variates in this data set, which means the variables in the individual self-efficacy/organizational bottom-up approach set are related to the variables in perceived barriers to MAE reporting. In the first significant pair of canonical variates, lower knowledge, intuitive decision-making, and

Table 3 Correlations among Knowledge about Medication Administration, Clinical Decision-Making, Nurse -physician Collaboration Satisfaction, and Perceived Barriers to Reporting Medication Administration Errors

\begin{tabular}{|c|c|c|c|c|c|c|c|}
\hline \multirow[t]{4}{*}{ Variables } & \multirow{2}{*}{\multicolumn{3}{|c|}{$\begin{array}{l}\text { Individual self-efficacy/Organizational } \\
\text { bottom-up approach set }\end{array}$}} & \multicolumn{4}{|l|}{$(N=218)$} \\
\hline & & & & \multicolumn{4}{|c|}{$\begin{array}{l}\text { Perceived barriers to reporting } \\
\text { medication administration errors }\end{array}$} \\
\hline & \multicolumn{7}{|l|}{$r(p)$} \\
\hline & 1 & 2 & 3 & 4 & 5 & 6 & 7 \\
\hline \multicolumn{8}{|c|}{ Individual self-efficacy/ Organizational bottom-up approach } \\
\hline 1. Knowledge about medication administration & 1.00 & & & & & & \\
\hline 2. Clinical decision-making & $-0.01(.868)$ & 1.00 & & & & & \\
\hline 3. Nurse-physician collaboration satisfaction & $-0.11(.114)$ & $-0.06(.348)$ & 1.00 & & & & \\
\hline \multicolumn{8}{|c|}{ Perceived barriers to reporting medication administration errors } \\
\hline 4. Fear & $0.08(.234)$ & $0.04(.544)$ & $-0.11(.099)$ & 1.00 & & & \\
\hline 5. Disagreement over medication error & $-0.24(<.001)$ & $0.16(.019)$ & $-0.04(.548)$ & $0.20(.004)$ & 1.00 & & \\
\hline 6. Reporting effort & $0.04(.581)$ & $-0.04(.532)$ & $-0.16(.019)$ & $0.25(<.001)$ & $0.43(<.001)$ & 1.00 & \\
\hline 7. Administrative responses & $0.04(.595)$ & $0.11(.102)$ & $-0.22(.001)$ & $0.52(<.001)$ & $0.40(<.001)$ & $0.27(<.001)$ & 1.00 \\
\hline
\end{tabular}


Table 4 Canonical Correlations between Individual Self-efficacy/ Organizational Bottom-up Approach set and Perceived Barriers to Reporting Medication Administration Errors

\begin{tabular}{lll}
\hline Variables sets & \multicolumn{2}{c}{$(N=218)$} \\
\cline { 2 - 3 } & \multicolumn{2}{c}{ Canonical variates } \\
\cline { 2 - 3 } Set 1: Individual self-efficacy/Organizational bottom-up approach \\
Knowledge about medication administration & -.83 & .12 \\
Clinical decision-making & .41 & -.57 \\
Nurse -physician collaboration satisfaction & .42 & .84 \\
\% Redundancy $\left(r^{2}\right)$ & 4.58 & 1.83 \\
Set 2: Perceived barriers to reporting medication & & \\
administration errors & & \\
Administrative responses & -.22 & -.94 \\
Disagreement over medication error & .64 & -.67 \\
Reporting effort & -.29 & -.46 \\
Fear & -.25 & -.42 \\
\% Redundancy $\left(r^{2}\right)$ & 2.02 & 2.24 \\
Canonical correlation & 0.37 & 0.23 \\
Significance test: $F(p)$ & $3.84(<.001)$ & $2.34(.031)$ \\
Variance explained & 13.4 & 5.3 \\
\hline
\end{tabular}

good nurse-physician collaboration satisfaction were associated with a higher perception of disagreement over medication errors. Decision-making is based on how information is organized in memory and the weight given to the information, and insufficient knowledge might be related to more intuitive decision-making [15]. Intuitive decision-makers show rapid information processing, simultaneous cue use, and evaluation cue use, because this type of decision-making is based on immediate apprehension of relevant situations [39]. Since nurses use an intuitive approach in data collection and an analytic approach in data processing and the identification of problems [40], a mismatch between decision-making style and situation is present. Intuitive decision-makers cannot make decisions based on their insufficient errorrelated knowledge; therefore, nurses' decision-making style may be transformed from intuitive to analytical. Supportive social and institutional contexts such as good collaboration satisfaction play an important role in decisionmaking [41]. In these conditions, when opportunities for discussion are provided, which may or may not be appropriate, intuitive decision-making would be fostered. To increase the precision of our understanding of their relationship, additional research needs to be conducted.

In the second pair, analytic decision-making and higher nurse-physician collaboration were associated with lower perceived barriers to error reporting. In clinical situations, smooth communication and cooperation not only enhance perceptions of being a team, lead to positive patient outcomes, and limit MAEs, but also promote informal error reporting among colleagues [6], and foster collaboration in decision-making [42]. In a previous study, when nurses were provided with potential error-driven situations, they would informally report errors as a means of clarifying and validating their opinion with their colleagues [6]. In addition, because formal error reporting is also regarded as a form of bottom-up communication [43], we suggest that staff-led communication and better teamwork must be established for better informal and formal error reporting. Analytical decision-making also needs to be carried out by healthcare professionals to reduce perception of barriers to reporting MAEs [15]. One strategy for supporting analytical decision-making may be the development of a clinical decision-making supporting system (CDSS) for error reporting. A CDSS is a computerized system that uses case-based reasoning rooted in treatment guidelines to assist clinicians in clinical decision-making [44]. It can improve medication safety by improving the process of care, such as by reducing medication errors and improving patient outcomes [45]. It is essential to use consistent terms [46, 47], such as those provided by a standardized taxonomy, when adopting a CDSS in error-reporting. It appears likely that a CDSS based on a medication-error taxonomy would facilitate analytic decision-making for error -reporting.

Better knowledge about medication administration and good nurse-physician collaboration satisfaction were the stronger factors in each significant pair of canonical variates for reducing perceived barriers to MAE reporting. According to a literature review, nurses' use of clinical reasoning to maintain safe medication administration was inadequate and clinical reasoning required in-depth knowledge [48]. Based on this study, knowledge about medication administration will not only be used in clinical reasoning for safe medication, but also as a precursor of error reporting. However, it is noteworthy that perception of disagreement over medication errors was the lowest barrier, even though the level of knowledge in this study was not high. Cognitive dissonance between perceived and real knowledge level is a possible reason. According to metacognition theory, if learners can accurately perceive the status of their knowledge, they will tend to think of themselves as good learners and complete work more efficiently [49]. Therefore, increased metacognition for knowledge of medication administration is needed.

A top-down approach with leadership from senior personnel takes time to create better patient-safety [50] and needs to be converted into a bottom-up approach to foster teambuilding and an open, fair environment. Better collaboration has been linked specifically to safetyrelevant performance [51] such as fewer hospitalacquired infections [52]. According to a recent study, nurses perceive physicians not as team members, but as 
independent healthcare workers [21]. In addition, nurses who perceive more respect perceive higher levels of nurse-physician collaboration [21]. When nurses work in an environment that supports and recognizes their professional role, they may feel respected, and therefore more confident participating in decision-making during the collaboration process [53] It is helpful if hospital administrators create management regulations for collaborative nurse-physician decision-making and to maximize nurses' involvement in the decision-making process [21]. However, self-help efforts for respectful communication between healthcare providers, which may be the foundation for collaboration, are even more important. Therefore, to improve nurse-physician communication, communication tools such as SBAR and cross-training/interprofessional educational programs would be helpful [53].

\section{Limitations}

Although this study provides meaningful results and findings, there are several limitations. A convenient sampling may suggest possible sampling bias; therefore, generalizability of the findings of this study to other settings is limited. Another important limitation is the relatively low reliability of medication administration knowledge, which means a low proportion of true variability to the total obtained variability. Therefore, instrument refinement and additional studies are necessary.

\section{Conclusions/implications for practice}

Despite these limitations, this study showed the possible influence of an individual self-efficacy/organizational bottom-up approach on reducing perceived barriers to MAE reporting. The findings suggest three recommendations for the clinical and policy phases. In the clinical phase, medication administration education, including high-alert drug administration training for nurses, may reduce disagreement about whether errors have occurred. In addition, a bottom-up approach strategy such as improving collaboration with interactive communication may facilitate MAE reporting. In the policy phase, administrators need to make an effort to promote analytical decision-making for reporting MAEs. Hospital leaders who construct and implement evidence-based medication error reporting systems that support decision-making can reduce the perceived barriers to reporting MAEs. The present study sheds light on the broader view of reducing perceived barriers to reporting MAEs and suggests further research that examines the effectiveness of the experimental study to extend the strategy of enhancing MAE reporting.

\section{Abbreviations}

ANOVA: Analysis of Variance; CDSS: Clinical Decision-making Supporting System; CVI: Content Validity Index; IRB: Institutional Review Board; KR: Kuder-
Richardson; MAE: Medication Administration Error; RN: Registered Nurse; UK: United Kingdom; US: United States

\section{Acknowledgements}

Not applicable.

\section{Authors' contributions}

All authors contributed to the conception and design of this study. CHK conducted the data analysis. MSK contributed to the interpretation of data, drafting the article and final approval of the version to be submitted. All authors worked on the revisions of the manuscript, read and approved the final manuscript.

\section{Funding}

This work was supported by the Dong-A University research fund.

\section{Availability of data and materials}

The datasets generated and/or analysed during the current study are not publicly available due but are available from the corresponding author on reasonable request.

\section{Ethics approval and consent to participate}

This study received ethics approval from the Institutional Review Board of Pusan National University Hospital in Korea. Written informed consent was obtained from each participant when survey were conducted.

\section{Consent for publication}

Not applicable.

\section{Competing interests}

The authors declare that they have no competing interests.

\section{Author details}

'Department of Nursing, Pukyong National University, 599-1, Daeyeon 3 dong, Namgu, Busan 48513, South Korea. ${ }^{2}$ College of Medicine, Dong-A University Hospital, 26 Daesingongwon-ro, Seo-gu, Busan 49201, South Korea.

Received: 20 December 2018 Accepted: 28 May 2019 Published online: 16 July 2019

\section{References}

1. Riga M, Vozikis A, Pollalis Y, Souliotis K. MERIS (medical error reporting information system) as an innovative patient safety intervention: a health policy perspective. Health Policy. 2015;119(4):539-48. https://doi.org/10. 1016/j.healthpol.2014.12.006.

2. Haw C, Stubbs J, Dickens GL. Barriers to the reporting of medication administration errors and near misses: an interview study of nurses at a psychiatric hospital. J Psychiatr Ment Health Nurs. 2014;21 (9):797-805. https://doi.org/10.1111/jpm.12143.

3. Yung HP, Yu S, Chu C, Hou IC, Tang FI. Nurses' attitudes and perceived barriers to the reporting of medication administration errors. J Nurs Manag. 2016;24(5):580-8. https://doi.org/10.1111/jonm.12360.

4. Bolye TA, Mahaffey T, MacKinnon NJ, Deal H, Hallstrom LK, Morgan H. Determinants of medication incident reporting, recovery, and learning in community pharmacies: a conceptual model. Res Social Adm pharm. 2011; 7(1):93-107. https://doi.org/10.1016/j.sapharm.2009.12.001.

5. Abdel-Latif MM. Knowledge of healthcare professionals about medication errors in hospitals. J Basic Clinical Pharmacy. 2016;7(3):87-92. https://doi.org/ 10.4103/0976-0105.183264.

6. Espin S, Wickson-Griffiths A, Wilson M, Lingard L. To report or not to report: a descriptive study exploring ICU nurses' perceptions of error and error reporting. Intensive Crit Care Nurs. 2010;26(1):1-9.

7. Sulosaari $V$, Suhonen $R$, Leino-Kilpi $H$. An integrative review of the literature on registered nurses' medication competence. J Clin Nurs. 2011;20(3-4): 464-78. https://doi.org/10.1111/j.1365-2702.2010.03228.x.

8. Hutchinson AM, Sales AE, Brotto V, Bucknall TK. Implementation of an audit with feedback knowledge translation intervention to promote medication error reporting in health care: a protocol. Implement Sci. 2015;10:70. https:// doi.org/10.1186/s13012-015-0260-y. 
9. Härkänen M, Vehviläinen-Julkunen K, Murrells T, Rafferty AM, Franklin BD. Medication administration errors and mortality: incidents reported in England and Wales between 2007-2016. Res Social Adm Pharm. 2018; In press. https://doi.org/10.1016/j.sapharm.2018.11.010.

10. Institute for Safe Medication Practices (ISMP). ISMP list of high-alert medications in acute carea settings. 2017. https://www.ismp.org/sites/default/ files/attachments/2018-08/highAlert2018-Acute-Final.pdf. Accessed 1 Mar 2019.

11. Saedder EA, Brock B, Nielsen NP, Bonnerup DK, Lisby M. Identifying high-risk medication: a systematic literature review. Eur J Clin Pharmacol. 2014;70(6): 637-645.

12. Hsaio GY, Chen IJ, Yu S, Wei IL, Fang YY, Tang FI. Nurses' knowledge of high-alert medications: instrument development and validation. J Adv Nurs. 2010;66(1):177-90. https://doi.org/10.1111/j.1365-2648.2009.05164.x.

13. Bjørk IT, Hamilton GA. Clinical decision making of nurses working in hospital settings. Nurs Res and Pract. 2011;2011:524918. https://doi.org/10.1155/ 2011/524918.

14. Hammond KR. Human judgment and social policy: irreducible uncertainty, inevitable error, unavoidable injustice. New York: OXFORD University Press; 1996.

15. Lauri S, Salanterä S. Developing an instrument to measure and describe clinical decision making in different nursing fields. J Prof Nurs. 2002;18(2): 93-100.

16. Murray M, Sundin D, Cope V. The nexus of nursing leadership and a culture of safer patient care. J Clin Nurs. 2018;27(5-6):1287-93. https://doi.org/10. 1111/jocn.13980.

17. Castel ES, Ginsburg LR, Zaheer S, Tamim H. Understanding nurses' and physicians' fear of repercussions for reporting errors: clinician characteristics, organization demographics, or leadership factors? BMC Health Serv Res. 2015;15:326. https://doi.org/10.1186/s12913-015-0987-9.

18. Aydon L, Hauck Y, Zimmer M, Murdoch J. Factors influencing a nurse's decision to question medication administration in a neonatal clinical care unit. J Clin Nurs. 2016;25(17-18):2468-77. https://doi.org/10.1111/jocn.13277.

19. Wagner LM, Damianakis T, Pho L, Tourangeau A. Barriers and facilitators to communicating nursing errors in long-term care settings. J Patient Saf. 2013;9(1):1-7. https://doi.org/10.1097/PTS.0b013e3182699919.

20. Parker D. Managing risk in healthcare: understanding your safety culture using the Manchester Patient Safety Framework (MaPSaF). J Nurs Manage. 2009;17(2):218-22.

21. Wang Y, Wan Q, Guo J, Jin X, Zhou W, Feng X, Shang S. The influence of effective communication, perceived respect and willingness to collaborate on nurses' perceptions of nurse-physician collaboration in China. App Nurs Res. 2018:41:73-9.

22. Guttman OT, Lazzara EH, Keebler JR, Webster KLW, Gisick LM, Baker AL Dissecting communication barriers in healthcare: a path to enhancing communication resiliency, reliability, and patient safety. J Patient Saf. 2018. https://doi.org/10.1097/PTS.0000000000000541. [Epub ahead of print]

23. Offredy $M$, Kendall S, Goodman C. The use of cognitive continuum theory and patient scenarios to explore nurse prescribers' pharmacological knowledge and decision-making. Int J Nurs Stud. 2008;45(6):855-68.

24. Evans SM, Berry JG, Smith BJ, Esterman A, Selim P, O'Shaughnessy J, DeVit M. Attitude and barriers to incident reporting: a collaborative hospital study. Qual Saf health care, vol. 15; 2006. p. 39-43.

25. Tabachnick BG, Fidell LS. Using multivariate statistics. 6th ed. Boston: Allyn \& Bacon; 2013.

26. Kim MS, Jung HK. Correlation among the medication error risk of high-alert medication, attitudes to single checking medication, and medication safety activities of nurses in the intensive care unit. J Kor Crit Care Nurs. 2015;8(1):1-10.

27. Kim HJ, Hur J. SPSS WIN 21.0 statistical analysis and interpretation. Seoul: Top books; 2016

28. Kim MS, Jung HK. Validity of Korean version of clinical decision making short form scale. Korean J Adult Nurs. 2014:26(4):403-12.

29. Baggs JG, Schmitt MH, Mushlin Al, Eldredge DH, Oakes D, Hutson AD. Nurse-physician collaboration and satisfaction with the decision making process in three critical care units. Am J Crit Care. 1997;6(5):393-9.

30. Lynn MR. Determination and quantification of content validity. Nurs Res. 1986;35(6):382-5.

31. Polit DF, Beck CT. The content validity index: are you sure you know what's being reported? Critique and recommendations. Res Nurs Health. 2006;29: 489-97.

32. Wakefield DS, Wakefield BJ, Uden-Holman T, Blegen MA. Perceived barriers in reporting medication administration errors. Best Pract benchmarking Healthc. 1996;1(4):191-7.
33. Brislin WR. Back-translation for cross-cultural research. J Cross-Cult Psychol. 1973:1:185e216. https://doi.org/10.1177/135910457000100301.

34. Kaiser H. An index of factor simplicity. Psychometrika. 1974;39:31-6.

35. Chiang HY, Lin SY, Hsu SC, Ma SC. Factors determining hospital nurses' failures in reporting medication errors in Taiwan. Nurs Outlook. 2010;58(1):17-25.

36. Lee E. Reporting of medication administration errors by nurses in south Korean hospitals. Int J Qual Health Care. 2017;29(5):728-34. https://doi.org/ 10.1093/intqhc/mzx096.

37. Oetzel JG, Ting-Toomey S. Face concerns in interpersonal conflict: a crosscultural empirical test of the face negotiation theory. Commun Res. 2003; 30(6):599-624.

38. Holmstrom AR, Airaksinen M, Weiss M, Wuliji T, Chan XH, Laaksonen R. National and local medication error reporting systems: a survey of practices in 16 countries. J Patient Saf. 2012;8(4):165-76. https://doi.org/10.1097/PTS. 0b013e3182676cf3.

39. Benner P, Tanner C. Clinical judgement: how expert nurses use intuition. Am J Nurs. 1987:87(1):23-31.

40. Lauri S, Salanterä S, Gilje FL, Klose P. Decision making of psychiatric nurses in Finland, Northern Ireland, and the United States. J Prof Nurs. 1999;43(5):369-70.

41. Espin S, Regehr G, Levinson W, Baker GR, Biancucci C, Lingard L. Factors influencing perioperative nurses' error reporting preferences. AORN J. 2007; 85(3):527-30.

42. Ghahramanian A, Rezaei T, Abdullahzadeh F, Sheikhalipour Z, Dianat I. Quality of healthcare services and its relationship with patient safety culture and nurse-physician professional communication. Health Promot Perspect. 2017:7(3):168-74. https://doi.org/10.15171/hpp.2017.30.

43. Hwang Jl, Ahn J. Teamwork and clinical error reporting among nurses in Korean hospitals. Asian Nurs Res. 2015;9(1):14-20. https://doi.org/10.1016/j. anr.2014.09.002.

44. de Vries AE, van der Wal MH, Nieuwenhuis MM, de Jong RM, van Dijk RB. Jaarsma $T$,... Jorna RJ. Perceived barriers of heart failure nurses and cardiologists in using clinical decision support systems in the treatment of heart failure patients. BMC Med Inform Decis Mak. 2013;26:54. https://doi. org/10.1186/1472-6947-13-54.

45. Jia $P$, Zhang $L$, Chen J, Zhao P, Zhang $M$. The effects of clinical decision support systems on medication safety: an overview. PLoS One. 2016;11(12): e0167683. https://doi.org/10.1371/journal.pone.0167683.

46. Taib IA, McIntosh AS, Caponecchia C, Baysari MT. A review of medical error taxonomies: a human factors perspective. Saf Sci. 2011;49(5):607-15. https:// doi.org/10.1016/j.ssci.2010.12.014.

47. De Wit HA, Hurkens KP, Mestres Gonzalvo C, Smid M, Sipers W, Winkens B, Mulder WJ, Janknegt R, Verhey FR, van der Kuy PH, Schols JM. The support o medication review in hospitalized patients using a clinical decision support system. Sprinerplus. 2016;5(1):871. https://doi.org/10.1186/s40064-016-2376-1.

48. Rohde E, Domm E. Nurses' clinical reasoning practices that support safety medication administration: an integrative review of the literature. J Clin Nurs. 2018;27(3-4):e402-11.

49. Ferrer-Torregrosa J, Jiménez-Rodríguez MÁ, Torralba-Estelles J, GarzónFarinós F, Pérez-Bermejo M, Fernández-Ehrling N. Distance learning ects and flipped classroom in the anatomy learning: comparative study of the use of augmented reality, video and notes. BMC Med Educ. 2016;16(1):230. https:// doi.org/10.1186/s12909-016-0757-3.

50. Stewart GL, Manges KA, Ward MM. Empowering sustained patient safety: the benefits of combining top-down and bottom-up approaches. J Nurs Care Qual. 2015;30(3):240-6. https://doi.org/10.1097/NCQ.0000000000000103.

51. Härkänen $M$, Turunen $H$, Vehviläinen-Julkunen K. Differences between methods of detecting medication errors: a secondary analysis of medication administration errors using incident reports, the global trigger tool method, and observations. https:// doi.org/10/1097/PTS.000000000000000261. Accessed 4 Nov 2018.

52. Boev C, Xia Y. Nurse-physician collaboration and hospital-acquired infections in critical care. Crit Care Nurse. 2015;35(2):66-72. https://doi.org/10.4037/ccn2015809.

53. Regan S, Laschinger HK, Wong CA. The influence of empowerment, authentic leadership, and professional practice environments on nurses' perceived interprofessional collaboration. J Nurs Manag. 2016;24(1):E54-61.

\section{Publisher's Note}

Springer Nature remains neutral with regard to jurisdictional claims in published maps and institutional affiliations. 